\title{
Propuesta de una metodología de análisis de la calidad global de la alimentación
}

\author{
Anna Christina Pinheiro $\mathrm{F}^{1 \mathrm{a}}$, Eduardo Atalah $\mathrm{S}^{2}$. \\ Proposal of a method to assess \\ global quality of diet
}

Background: Several studies have attempted to assess the global quality of diet. One of such initiatives is the Healthy Eating index (HEI), developed in the Unites States. However, its application in other countries requires certain adaptations. Aim: To adapt the HEI to the Chilean population and to assess its value in Chilean school age children and adults. Ma terial anal methods: A random sample of 264 school age children aged $13.4 \pm 0.7$ years and 264 adults aged $35.7 \pm 0.6$ years was studied. A 24 hours food recall was done and the HEI seas calculated considering 10 variables based in the Feeding Guides for the Chilean population. Feeding habits were classified as healthy when the score was over 80 (of a maximum of 100), as requiring changes when the score was between 51 and 80 and as unhealthy when the score was 50 or less. Results: The mean scores for children and adults were $58.4 \pm 11.3$ and $56.2 \pm 11.1$ respectively $(\mathrm{p}<0.02)$. The variables with lower scores were vegetables, fruits, dairy products and sodium. Only $1.5 \%$ of the population had healthy feeding habits, without differences by sex or age. Feeding quality was significantly worse among obese subjects. Conclusions: The HEI is a tool that can be used in the Chilean population and can be used to assess the effectiveness of health promotion initiatives. It also can help to focalize educational programs to specific nutrients (Rev Méd Chile 2005; 133: 175-82).

(Key words: Behavior and behavior mechanisms; Diet; Food habits)

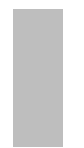

Recibido el 20 de abril 2004. Aceptado en versión corregida el 30 de noviembre 2004.

${ }^{1}$ Escuela de Nutrición, Facultad de Medicina, Universidad de Chile. ${ }^{2}$ Departamento de Nutrición, Facultad de Medicina, Universidad de Chile.

aNutricionista MSc.

$\mathrm{N}$ umerosos estudios epidemiológicos han demostrado un efecto protector o un mayor riesgo de enfermedades crónico degenerativas (ECD) asociado al consumo de determinados alimentos o nutrientes. La Organización Mundial de la Salud (OMS) ha señalado que unos pocos

Correspondencia a: Anna Christina Pinheiro Fernandes. Escuela de Nutrición, Facultad de Medicina, Universidad de Chile. Av. Independencia, 1027. Clasificador 7, Independencia, Santiago, Chile. Fax: (56-2) 6786182. E-mail: apinheiro@med.uchile.cl factores de riesgo son responsables de gran parte de la carga de enfermedad ${ }^{1-4}$. En los países con mayor nivel de desarrollo, la hipertensión arterial, hipercolesterolemia, bajo consumo de frutas y verduras, el exceso de peso, la falta de actividad física y el tabaquismo son responsables de más de un tercio de los años de vida perdidos por muerte o discapacidad, cinco de ellos estrechamente asociados a la alimentación ${ }^{3,4}$.

El desarrollo de las Guías Alimentarias y de la Pirámide Alimentaria para la población chilena fue 
la estrategia utilizada por el Ministerio de Salud en 1997, con el propósito de promover estilos de vida saludables y orientar a la población en la selección de alimentos más saludables que reduzcan el riesgo de ECD ${ }^{5-8}$. Ello implica el desafío de lograr cambios positivos en las conductas alimentarias y de disponer mecanismos que permitan monitorear y evaluar los cambios obtenidos.

En la literatura se han descrito diversas metodologías que analizan de manera global la calidad de la alimentación de una población ${ }^{9-15}$. Entre ellas, destaca el Healthy Eating Index (HEI), que fue desarrollado por el Centro para la Promoción de la Nutrición del Departamento de Agricultura de Estados Unidos en 1995, con el objetivo de determinar el grado de adhesión de la población norteamericana a las Guías Alimentarias. El HEI se basa en datos obtenidos en encuestas alimentarias, a partir de los cuales se construyen 10 variables, cada una con un puntaje que puede fluctuar de 0 a 10. La suma de los puntajes posibilita la construcción de un indicador con un valor máximo de 100 puntos y la clasificación de la alimentación en 3 categorías: saludable, necesita cambios, poco saludable.

Desde la implementación de las Guías Alimentarias y de la Pirámide Alimentaria, se han realizado limitados estudios que analicen su grado de cumplimiento en la población chilena ${ }^{16-18}$. Tampoco se ha desarrollado una metodología que permita evaluar en forma integral la calidad de la dieta, monitorear su evolución en el tiempo y el posible impacto de las actividades de promoción de la salud que se han realizado.

Considerando la metodología utilizada en la elaboración del HEI norteamericano, hemos desarrollado el Índice de Alimentación Saludable (IAS), que analiza el grado de cumplimiento de las Guías Alimentarias y las recomendaciones de ingesta de porciones de alimentos de acuerdo a la Pirámide Alimentaria chilena. El propósito de este estudio es adaptar la metodología del HEI a las recomendaciones para la población chilena y aplicarlo a un grupo de escolares y adultos de tres ciudades.

\section{MATERIAL Y MÉTODO}

Para determinar el tamaño de muestra se estimó que $10 \%$ de la población presentaría una alimen- tación saludable (IAS $\geq 80$ ), lo que al considerar $4 \%$ de error y un nivel de confianza de 95\%, implicó estudiar a 216 personas en cada grupo de edad (adultos y escolares). El estudio se basó en una muestra aleatoria, representativa, trietápica de 264 escolares y 272 adultos de tres ciudades de Chile (Santiago, Antofagasta y Temuco) de un proyecto orientado a evaluar la exposición humana al arsénico ${ }^{19}$.

Para determinar la muestra de adultos, fueron seleccionadas todas las empresas afiliadas a la Asociación Chilena de Seguridad (ACHS) en esas tres ciudades, en las cuales hubiera más de 10 afiliados. Por medio de un sorteo aleatorio, fueron seleccionadas 10 empresas en Antofagasta y Temuco y 16 en Santiago, sorteándose posteriormente 7 personas en cada una de ellas. Para la muestra de escolares, fueron identificados todos los colegios municipalizados y particular subvencionados que registraran existencia del $8^{\underline{0}}$ año básico, seleccionándose aleatoriamente 10 en Temuco y Antofagasta y 20 en Santiago. En cada escuela, se sortearon aleatoriamente 7 niños en Antofagasta y Temuco y 5 a 6 en Santiago.

A cada participante se le aplicó una encuesta alimentaria de recordatorio del día anterior en los respectivos locales de trabajo o estudio. Las encuestas fueron realizadas por una nutricionista previamente entrenada, con el apoyo de material fotográfico que representaba los utensilios domésticos de uso habitual en la alimentación (distintos tipos de platos, tazas y cucharas). La composición nutricional de los alimentos fue calculada utilizando el software Sofnut, $2002^{20}$, que entrega, además, información respecto a las porciones consumidas de acuerdo a las recomendaciones de la Pirámide Alimentaría chilena.

Personal entrenado obtuvo el peso y la talla en condiciones estandarizadas, utilizando balanzas electrónicas portátiles calibradas con precisión de 100 gramos y un cartabón portátil con precisión de 0,1 centímetros. Se calculó el Índice de Masa Corporal $\left(\mathrm{Kg} / \mathrm{m}^{2}\right)$ y se clasificó según normas del Ministerio de Salud. En escolares se analizó Índice de Masa Corporal (IMC) según edad y sexo con relación a las tablas del $\mathrm{CDC} / \mathrm{NCHS}$ y se clasificó según el siguiente criterio: bajo peso $(<$ percentil $10)$, normal (entre percentil 10 y 84), sobrepeso (entre percentil 85 y 94) y obesidad ( $\geq$ percentil $95)^{21}$. En los adultos se consideraron los siguien- 
tes criterios: bajo peso (IMC < 18,5), normal (entre 18,5 y 24,9), sobrepeso (entre 25,0 y 29,9) y obesidad $(\geq 30,0 \mathrm{~kg} / \mathrm{m})^{22}$.

Para el cálculo del Indice de Alimentación Saludable (IAS) se utilizaron 10 variables. Cinco de ellas se refieren a las porciones consumidas de los principales grupos de alimentos de la pirámide alimentaria (cereales, verduras, frutas, lácteos, carnes), cuatro a las metas alimentarias (consumo de lípidos totales, grasas saturadas, azúcares y sodio) y una se relaciona con el grado de variedad en el consumo de alimentos. Cada componente recibe un puntaje que varía de 0 a 10 de acuerdo a los criterios establecidos en la Tabla 1, dónde 10 significa el cumplimiento de la meta propuesta por el Ministerio de Salud. En las variables lípidos totales (G\%), grasas saturadas y azúcar, se considera el porcentaje aportado por estos nutrientes con relación a las calorías totales. En la variable azúcar, considera el consumo de azúcar propiamente tal, más el aporte proveniente de la miel, mermeladas, bebidas gaseosas y jugo en polvo. La variedad se calcula considerando las porciones consumidas de cereales, carnes, verduras, lácteos y carnes. El IAS se calcula sumando el puntaje de las 10 variables, lo que permite un máximo teórico de 100 puntos. La clasificación de la alimentación se realiza de acuerdo al puntaje total y se divide en tres categorías: > 80 'Saludable'; 51-80 'Necesita cambios'; < 50 puntos: 'Poco saludable'.

Como en la encuesta alimentaria original no fue pesquisada la sal agregada a los alimentos, se estimó un aporte por esta vía de 2,4 g de sal/día, considerando los datos de un estudio nacional ${ }^{23}$.

Los datos fueron procesados con el programa SPSS, versión $10.0^{24}$. En el análisis de las variables con distribución normal se utilizaron promedios, desviación estándar y el test de ANOVA para la comparación entre los grupos. En las variables nominales y categóricas se utilizaron distribuciones de frecuencia y $\chi^{2}$. En todos los análisis se consideró significativo un valor de $\mathrm{p}<$ 0,05 .

\section{RESULTADOS}

El grupo estudiado estuvo conformado por 263 escolares y 265 adultos laboralmente activos, con una edad promedio de $13,4 \pm 0,7$ y $35,7 \pm 10,6$ años respectivamente y con una distribución homogénea por sexo en ambos grupos. Cerca de $40 \%$ de los encuestados presentaba algún grado de sobrepeso u obesidad según el IMC (Tabla 2), con una proporción significativamente mayor en los adultos $(\mathrm{p}<0,001)$.

Tabla 1. C riterios utilizados para definir el puntaje de cada variable del Índice de Alimentación Saludable

\begin{tabular}{|c|c|}
\hline Variable & Puntaje \\
\hline Cereales & 0: $\sin$ consumo; $10: \geq$ a $100 \%$ de las porciones recomendadas según sexo y edada \\
\hline Verduras & 0: sin consumo; $10: \geq$ a $100 \%$ de las porciones recomendadas según sexo y edada \\
\hline Frutas & 0: sin consumo; $10: \geq$ a $100 \%$ de las porciones recomendadas según sexo y edada \\
\hline Lácteos & 0: sin consumo; $10: \geq$ a $100 \%$ de las porciones recomendadas según sexo y edada \\
\hline Carnes & 0: sin consumo; $10: \geq$ a $100 \%$ de las porciones recomendadas según sexo y edada \\
\hline Grasa total & 0: $\mathrm{G} \%>45 \%$ o $<15 \% ; 10: \mathrm{G} \%$ entre $15 \%$ y $30 \% \mathrm{a}, \mathrm{b}$ \\
\hline Grasa saturada & $0:>15 \%$ de las calorías totales; $10:<10 \%$ de las calorías totales ${ }^{\mathrm{a}, \mathrm{b}}$ \\
\hline Azúcares & $0:>15 \%$ de las calorías totales; $10:<10 \%$ de las calorías totales ${ }^{\mathrm{a}, \mathrm{c}}$ \\
\hline Sodio & $0:>4,8 \mathrm{~g} /$ día; $10:<2,4 \mathrm{~g} /$ día $^{\mathrm{a}, \mathrm{c}}$ \\
\hline Variedad & $\begin{array}{l}0:<25 \% \text { de las porciones de cereales, carnes, verduras, lácteos y carnes; } 2: \geq \text { a } 25 \% \\
\text { de la porciones recomendadas de cada grupo a El puntaje global se obtiene sumando } \\
\text { las } 5 \text { variables }\end{array}$ \\
\hline
\end{tabular}

aLos valores intermedios son calculados de forma proporcional

bPuntos de corte de acuerdo a las RDA's/HEI ${ }^{15}$

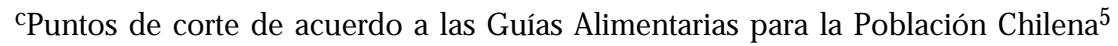


Tabla 2. Clasificación del estado nutricional en la muestra estudiada de acuerdo al IM C

\begin{tabular}{|lrrrrrr|}
\hline Clasificación del & \multicolumn{2}{c}{ Escolares } & \multicolumn{2}{c}{ Adultos } & \multicolumn{2}{c|}{ Total } \\
IMC & $\mathrm{n}$ & $\%$ & $\mathrm{n}$ & $\%$ & $\mathrm{n}$ & $\%$ \\
\hline Bajo peso & 5 & 1,9 & 2 & 0,8 & 7 & 1,3 \\
Normal & 177 & 67,3 & 121 & 45,7 & 298 & 56,4 \\
Sobrepeso & 55 & 20,9 & 104 & 39,2 & 159 & 30,1 \\
Obesidad & 26 & 9,9 & 38 & 14,3 & 64 & 12,1 \\
Total & 263 & 100,0 & 265 & 100,0 & 528 & 100,0 \\
\hline
\end{tabular}

$\chi^{2}: 29,15 p<0,01$

Tabla 3. Puntaje promedio de los componentes del IAS en los dos grupos estudiados

\begin{tabular}{|lccc|}
\hline Variable & $\begin{array}{c}\text { Escolares } \\
\text { Promedio } \pm \mathrm{DE}\end{array}$ & $\begin{array}{c}\text { Adultos } \\
\text { Promedio } \pm \mathrm{DE}\end{array}$ & $\begin{array}{c}\text { Total } \\
\text { Promedio } \pm \mathrm{DE}\end{array}$ \\
\hline Cereales & $7,8 \pm 2,3$ & $7,2 \pm 2,5$ & $7,5 \pm 2,41$ \\
Verduras & $3,5 \pm 3,2$ & $3,6 \pm 2,8$ & $3,6 \pm 3,0$ \\
Frutas & $2,9 \pm 3,7$ & $2,4 \pm 3,3$ & $2,6 \pm 3,5$ \\
Lácteos & $3,0 \pm 3,2$ & $2,5 \pm 3,5$ & $2,7 \pm 3,4$ \\
Carnes & $6,9 \pm 3,3$ & $7,0 \pm 3,2$ & $6,9 \pm 3,3$ \\
Lípidos totales & $8,5 \pm 2,8$ & $8,4 \pm 3,0$ & $8,4 \pm 3,0$ \\
Lípidos saturados & $8,6 \pm 2,9$ & $9,0 \pm 2,4$ & $8,8 \pm 2,7$ \\
Sodio & $3,1 \pm 2,0$ & $3,2 \pm 2,7$ & $3,2 \pm 2,7$ \\
Azúcar & $7,8 \pm 3,6$ & $6,9 \pm 4,2$ & $7,3 \pm 4,01$ \\
Variedad & $6,2 \pm 2,0$ & $6,0 \pm 2,0$ & $6,1 \pm 2,02$ \\
Total & $58,4 \pm 11,3$ & $56,2 \pm 11,1$ & $57,3 \pm 112$ \\
\hline
\end{tabular}

${ }^{1} \mathrm{p}<0,05 ;{ }^{2} \mathrm{p}<0,02$

T ABLA 4. Clasificación del IAS según grupo de edad

\begin{tabular}{|lccc|}
\hline IAS & $\begin{array}{c}\text { Escolares } \\
\mathrm{n}=203 \\
\%\end{array}$ & $\begin{array}{c}\text { Adultos } \\
\mathrm{n}=265 \\
\%\end{array}$ & $\begin{array}{c}\text { Total } \\
\mathrm{n}=528 \\
\%\end{array}$ \\
\hline Saludable & 1,5 & 1,5 & 1,5 \\
Necesita de cambios & 69,4 & 63,1 & 66,2 \\
Poco saludable & 29,1 & 35,4 & 32,3 \\
Total & 100,0 & 100,0 & 100,0 \\
\hline
\end{tabular}

$\chi^{2}: 2,49 ; \mathrm{p} \mathrm{NS}$ 
Tabla 5. D istribución de la clasificación del IAS de acuerdo al Índice de M asa Corporal

\begin{tabular}{|c|c|c|c|}
\hline $\begin{array}{l}\text { Clasificación } \\
\text { del IAS }\end{array}$ & $\begin{array}{c}\text { Normal } \\
\mathrm{n}=298 \\
\%\end{array}$ & $\begin{array}{c}\text { Sobrepeso } \\
\mathrm{n}=159 \\
\%\end{array}$ & $\begin{array}{c}\text { Obesidad } \\
\mathrm{n}=64 \\
\%\end{array}$ \\
\hline Saludable & 1,7 & 1,3 & 1,6 \\
\hline Necesita de cambios & 71,5 & 64,2 & 59,4 \\
\hline Poco saludable & 26,8 & 34,6 & 39,1 \\
\hline Total & 100,0 & 100,0 & 100,0 \\
\hline
\end{tabular}

$\chi^{2}: 34,22 p<0,01$

El puntaje promedio del IAS en ambos grupos fue bajo $(57,3 \pm 11,3)$, lo que representa un cumplimiento inferior a $60 \%$ de las metas alimentarias (Tabla 3). Los puntajes más bajos correspondieron a verduras, frutas, lácteos y sodio y los más altos a grasas totales, grasas saturadas, cereales y carnes. La comparación de los puntajes entre escolares y adultos muestra diferencias significativas para cereales, azúcares, variedad y puntaje total, con mejores resultados en escolares.

Al clasificar el IAS según el puntaje total observamos que solamente una pequeña proporción del grupo tiene una alimentación considerada saludable (Tabla 4). Dos tercios de la muestra necesita realizar cambios en sus patrones alimentarios y el tercio restante cumple con menos de la mitad de las recomendaciones del Ministerio de Salud ('Poco saludable').

Al analizar el IAS según IMC (Tabla 5) se observa un aumento significativo de la proporción de personas en la categoría 'poco saludable' a medida que aumenta el exceso de peso $(p<0,01)$.

\section{DisCUSIÓN}

El desarrollo de las Guías Alimentarias tiene como principal objetivo transformar las recomendaciones de consumo de nutrientes en recomendaciones de consumo de alimentos, de modo tal que sea factible su entendimiento por parte de la población general ${ }^{25}$. A pesar de su implementación en 1997, son escasos los trabajos que evalúan el grado de cumplimiento o entendimiento de las Guías Alimentarias ${ }^{17,18}$.
La creación de metodologías que sean capaces de evaluar la calidad de alimentación de la población ha sido tema de constantes investigaciones llevadas a cabo principalmente en Norteamérica. El HEI desarrollado en 1995 por el Centro para la Promoción de la Nutrición del Departamento de Agricultura de Estados Unidos ha sido utilizado para monitorear la situación de la alimentación en muestras representativas de la población de ese país. El estudio más reciente fue realizado en el período 1999-2000 utilizando los datos de ingesta obtenidos del National Health and Nutrition Examination Survey ${ }^{15}$. En esta oportunidad, solamente $10 \%$ de la población tuvo su alimentación considerada como buena de acuerdo a la metodología utilizada para la construcción del índice. La gran mayonía (74\%) necesitaba realizar cambios en su conducta alimentaria con el fin de adecuarse a las recomendaciones de ingesta delimitadas por las Guías Alimentarias y Pirámide Alimentaria de este país.

Desde su primera aplicación, en 1989, el valor promedio del HEI ha aumentado en 2,3 puntos si se comparan con los resultados obtenidos en 2000. Entre las variables que mejoraron su puntaje se encuentran las grasas saturadas y la variedad. Los cambios pudieron ser atribuidos principalmente a la introducción de la pirámide y Guías Alimentarias para la Población norteamericana y a las intensas campañas educativas realizadas respecto a alimentación y nutrición ${ }^{26}$. Factores como género, edad, raza/etnia, lugar de nacimiento, educación e ingreso han sido asociados con los puntajes obtenidos en el índice.

La validez del HEI se ha demostrado con la utilización de metodologías sensibles como los 
biomarcadores plasmáticos ${ }^{27}$. Hann y colaboradores al estudiar la relación entre cáncer de mama y dieta, encuentran que los puntajes mas elevados del HEI se asocian a mayores concentraciones plasmáticas de b-caroteno, a-caroteno, b-cryptoxantina, luteína y vitamina C. A pesar de estas evidencias, han surgido propuestas para modificar la metodología del HEI con el objetivo de mejorar su capacidad predictiva con relación a enfermedades crónicas degenerativas ${ }^{15,28,29}$.

La utilización de la metodología del HEI para la población chilena obligó necesariamente a su adaptación a las recomendaciones del Ministerio de Salud para la ingesta de alimentos y nutrientes ${ }^{5,8}$, lo que se llevó a cabo en este estudio. No incluimos la variable colesterol presente en el índice original, ya que de cierto modo la variable grasa saturada es capaz de pesquisar la ingesta de este nutriente. Esta variable fue substituida por otra denominada azúcar, la cual tiene como finalidad evaluar el consumo de azúcar simple, otro nutriente critico definido por el Ministerio de Salud.

Los cambios realizados en la metodología del HEI conforman lo que hemos llamado Índice de Alimentación Saludable (IAS) ${ }^{30}$. La construcción del indicador estuvo marcada por varias limitaciones, las cuales estuvieron asociadas principalmente a la dificultad en la obtención de datos confiables de ingesta alimentaria ${ }^{31}$. La encuesta utilizada no contemplaba la pesquisa de la sal agregada a las preparaciones, lo que obligó a estimarla considerando datos de consumo promedio de sal en la población chilena ${ }^{23}$.

En nuestros resultados sorprende los adecuados puntajes obtenidos para grasas totales y saturadas, sugiriendo que un alto consumo no sería un problema frecuente en la población estudiada. Nuestra impresión es que este hecho podría deberse en parte a un subregistro en la pesquisa del aceite agregado a las preparaciones. Se requiere mayor información para aclarar los verdaderos niveles de consumo de grasa en la población chilena, cuyo consumo aparente según las hojas de balance de FAO ha crecido en $34 \%$ en los últimos 10 años. Si se hiciera una corrección de la variable grasas totales y saturadas el puntaje promedio del IAS sería aún más bajo.
Una vez más, se demuestra que los alimentos más críticos por su bajo consumo son verduras, frutas y lácteos, que en promedio representa un tercio de lo recomendado por el Ministerio de Salud ${ }^{32}$. Aparentemente los esfuerzos de educación alimentaria no han logrado modificar esta situación y debiera ser una prioridad en las políticas de salud. Otro nutriente crítico es el sodio, en este caso por exceso de consumo, con una ingesta promedio estimada en $4,6 \pm 1,1$ gramos por persona. Como este valor se basó en parte en estimaciones de la sal agregada, podría haber un margen importante de error y necesita ser validado por metodologías más precisas, incluyendo por ejemplo la excreción urinaria de sodio.

En los resultados destaca un bajo puntaje promedio, independientemente del género, edad, o ubicación geográfica. Al analizar los puntajes del IAS de acuerdo al IMC, los resultados reflejan los patrones alimentarios descritos en la literatura, dónde la mayor parte de los individuos con exceso de peso presentan una alimentación Poco saludable o Necesita realizar cambios en su alimentación. Ello demuestra además que la población estudiada presenta patrones de consumo muy semejantes y que un IMC normal no refleja necesariamente una alimentación saludable. También obliga a reevaluar los puntos de corte para la clasificación del IAS, los que no tendrían porque ser coincidentes con los utilizados en el HEI. Estudios que analicen a la incidencia de enfermedades crónicas en función del IAS pueden contribuir a responder a esta interrogante.

Debido a la complejidad que involucra la construcción del IAS, no creemos que deba ser aplicado a nivel individual, aún con el apoyo de una rutina computacional. Su mayor utilidad radica en la estimación de la calidad de la alimentación comunitaria, posibilitando la adopción de estrategias de intervención en la población gene$\mathrm{ral} o$ focalizadas en determinados nutrientes y/o grupos de alimentos y su posterior evaluación secuencial. Esta es una verdadera prioridad nacional, considerando la creciente carga de enfermedad que deberemos enfrentar, la cual se encuentra directamente vinculada a nuestras inadecuadas prácticas de alimentación. 


\section{REFERENCIAS}

1. Ribol E, Norat T. Epidemiologic evidence of the protective effect of fruit and vegetables on cancer risk. Am J Clin Nutr 2003; 78: 559S-569S.

2. Hooper L, Bartlett C, Davey Smith G, Ebrahim s. Reduced dietary salt for prevention of cardiovascular disease. Cochrane Database Syst Rev. 2003; CD003656.

3. OMS. Estrategia mundial sobre régimen alimentario, actividad física y salud. A57/9. Ginebra, 2004.

4. OMS. Informe sobre la salud del mundo 2002: Reducir los riesgos y promover una vida sana. Ginebra, OMS, 2002.

5. Castilo C, Uauy R, Atalah E. (Eds.). Guías de alimentación para la población chilena. $1^{\mathrm{a}}$ ed. Imprenta La Nación, Santiago, 1997.

6. Castilo C, Uauy R, Atalah E. (Eds.). Guías de alimentación para el adulto mayor. Bases para la acción. 1ae ed. Imprenta La Nación, Santiago, 1999.

7. Burrows R, CastiLo C, Аtalah E, Uauy R. (Eds.). Guías de alimentación para la mujer chilena. 1aㅡ ed. Santiago: Imprenta La Nación, 2001.

8. Jury G, Urteaga C, Taibo M. Ponciones de intercambio de alimentos. En: Jury G, Urteaga C, Taibo $\mathrm{M}$, editores. Porciones de Intercambio y Composición Química de los Alimentos de la Pirámide Alimentaria Chilena. Santiago: LOM Ediciones, 1999; 3-12.

9. Kennedy, E T, Ohls J, Carlson S, Fleming K. The Healthy Eating Index: Design and aplications. J Am Diet Assoc 1996; 95: 1103-8.

10. Patterson R, Haines PS, Popkin BM. Dietary Quality Index: Capturing a multidimensional behavior. J Am Diet Assoc 1994; 94: 57-64.

11. Haines PS, Siega-Rzz AM, Popkin BM. The Diet Quality Index revised: a mesurement instrument for populations. J Am Diet Assoc 1999; 99: 697704.

12. KANT A K. Indexes of overall diet quality: a review. J Am Diet Assoc 1996; 96: 785-91.

13. Mcculough ML Feskanich D, Stampfer MJ, Giovannucci EL, Rimm EB, Hu FB, Sitegelman D, Hunter DJ, Colditz GA, WiLetT WC. Diet quality and major chronic disease risk in men and women: moving toward improved dietary guidance. Am J Clin Nutr 2002; 76: 1261-71.

14. The Healthy Eating Index: 1999-2000 (PDF). United States Department of Agriculture. Center for
Nutrition Policy and Promotion. Disponible en: http://www.usda.gov/cnpp/healthyeating.html [capturado en febrero de 2004].

15. NickLas T. Assessing diet quality in children and adolescents. J Am Diet Assoc. 2004; 104: 1383-4.

16. Domper A, Zacarias IA, Olivares SC, Hertrampf ED. Evaluación de un programa de información en nutrición al consumidor. Rev Chil Nutr 2003; 30: 43-51.

17. YÁñez R, Olunares SC, Torres IL, Guevara MN. Validación de las Guías y de la Pirámide Alimentaria en escolares de 5o a $8^{\circ}$ básico. Rev Chil Nutr 2000; 27: 358-67.

18. Araya M, Atalah E. Factores que determinan la selección de alimentos en familias de sectores populares. Rev Chil Nutr 2002; .29: 308-15.

19. CONAMA. Escuela de Salud Pública. Facultad de Medicina. Universidad de Chile. Análisis de Exposición Humana a Arsénico en Grandes Ciudades. Estudio 21-0022-002. Informe de Avance. Santiago, diciembre de 2000.

20. Pinheiro AC, Fuentes LA. Sofnut, versión 2002. Software para el procesamiento de datos de ingesta de alimentos. Sofmatica, Ltda.

21. Ministerio de Salud. Consejo Asesor de Nutrición. GRUPO DE EXPERTOS. Norma Técnica de evaluación nutricional del niño de 6 a 18 años. Rev Chil Nutr 2004; 2: 128-37.

22. OMS. Obesity: preventing and managing the global epidemic. Report of a WHO Consultation on Obesity. Geneva, 1997.

23. Durán FE, Soto AD, Asenjo IG, Labraña AM, Quiróz VG, PRADENAS FP. Ingesta dietaria de sodio, potasio y calcio en embarazadas normotensas. Rev Chil Nutr 2002; 29: 40-6.

24. SPSS for Windows, version 10.0. SPSS Inc 19891999.

25. Olivares S, Zacarías I. Bases para el desarrollo de Guías de Alimentación. In Castillo C, editor. Guías de Alimentación para la población Chilena. 1a ed. Diario La Nación, 1997: 11-21.

26. Eat 5 to 9 a day. National Cancer Institute. Disponible en: http://www.5aday.gov [capturado en marzo de 2003].

27. Hann CS, Rock CL, King I, Drewnowski. A Validation of the Healthy Eating Index with use of plasma biomarkers in a clinical sample of women. Am J Clin Nutr 2001; 74: 479-86. 
28. Mc Cullough ML, Feskanich D, Rimm EB, GiovanNucci EL, Ascherio A, Variyam JN et al. Adherence to the Dietary Guidelines for Americans and risk of major chronic disease in men. Am J Clin Nutr 2000; 72: 1223-31.

29. Mc Culough ML, Feskanich D, Stampfer MJ, Rosner BA, Hu FB, Hunter DJ ET AL. Adherence to the Dietary Guidelines for Americans and risk of major chronic disease in women. Am J Clin Nutr 2000; 72: 1214-22.
30. Pinheiro AC. Aplicación del Índice de Alimentación Saludable en un Grupo de Escolares y Adultos Chilenos [disertación]. Santiago: Universidad de Chile, Facultad de Medicina, 2002.

31. URTeaga C, Pinheiro Ac. Investigación alimentaria: consideraciones prácticas para mejorar la confiabilidad de los datos. Rev Chil Nutr 2003; 30: 235-42.

32. Urteaga C, Pinheiro Ac, Atalah E. Comparación de los resultados de dos métodos de encuestas alimentarias. Arch Latinoam Nutr 2003; 53: 172-7. 